\title{
Balkanologie
}

Balkanologie Revue d'études pluridisciplinaires

Vol. VII, $n^{\circ} 2 \mid 2003$

Volume VII Numéro 2

\section{Alice KRIEG-PLANQUE, «Purification ethnique ». Une formule et son histoire}

Paris : CNRS éditions, 2003, 528 p.

\section{Patrick Michels}

\section{CpenEdition}

\section{Journals}

Édition électronique

URL : http://journals.openedition.org/balkanologie/1969

DOI : 10.4000/balkanologie.1969

ISSN : 1965-0582

\section{Éditeur}

Association française d'études sur les Balkans (Afebalk)

Édition imprimée

Date de publication : 1 décembre 2003

Pagination : 231-233

ISSN : 1279-7952

\section{Référence électronique}

Patrick Michels, «Alice KRIEG-PLANQUE, "Purification ethnique ». Une formule et son histoire », Balkanologie [En ligne], Vol. VII, n² 2 | 2003, mis en ligne le 19 février 2009, consulté le 17 décembre 2020. URL : http://journals.openedition.org/balkanologie/1969; DOI : https://doi.org/10.4000/ balkanologie.1969 


\section{Krieg-Planque (Alice), \\ « Purification ethnique ». Une formule et son histoire, Paris : CNRS éditions, 2003, $528 \mathrm{p}$.}

Constitué des chapitres 3 et 6 de sa thèse soutenue à l'université de paris 13, en science du langage en 2000, ce travail d'analyse de discours ne s'intéresse pas aux revendications des acteurs, mais à qui en a rendu compte, par quel moyen, dans quel lieu.

Le corpus utilisé est constitué de la presse écrite (quotidiens, hebdomadaires, mensuels), extra-médiatique (revues, livres, bd, brochures, chansons, tracts).

A. Krieg-Planque se penche sur la fonction que remplit la formulation, l'utilisation stratégique des termes, le jeu des mots, le sens qu'ils portent, transportent, déportent. Elle rappelle qu'il existe plusieurs versions d'un événement (une seule est vraie, " l'auteur de la version fausse soit se trompe, soit ment ", p. 211).

L'auteur examine les 3 néologismes que sont " purification ", " nettoyage " et " épuration " ethnique, afin de voir s'il s'agit bien d'une " formule ", c'est-à-dire un " objet descriptible dans les catégories de la langue n (p. 14), objet tout à la fois dominant et perpétuellement questionné (p. 18), ou référant social (p. 20), autrement dit qui fait sens en soi.

Le substrat événementiel est constitué de 5 " épisodes " : la découverte des camps en été 1992 ; la découverte des viols en hiver 1992-1993; la décision du général Morillon de rester à Srebrenica, en mars-avril 1993 ; la chute d'un obus sur le marché de Sarajevo en février 1994 ; et la prise imminente de Gorazde en avril 1994. Ces cinq événements marquent des tournants dans les discours tenus (p. 31) : répétition du projet nazi ; problème social ; paroxysme du discours envahi par la mauvaise conscience et la culpabilité ; " réveil des consciences " ou non; polémique sur les faits.

Les trois néologismes (purification / nettoyage / épuration) sont " pour une majorité de locuteurs une seule et même formule " (p. 224) ; ils relèvent d'un même paradigme désignationnel. Son analyse de trois journaux entre 1980 et 1994 , le Nouvel observateur, l'Express et le Monde, montre que les trois variantes les plus utilisées et leur hiérarchie sont les mêmes : purification, nettoyage, épuration (p. 225). Mais leur apparition est récente, et s'est opérée dans le contexte du conflit yougoslave des années 1990 : en fait, la découverte des camps marque l'apparition de " purification ethnique " en tant que formule (p. 36). Les périodes de fort emploi de la formule coïncident avec des périodes de forte médiatisation du conflit yougoslave (p. 241).

La formule n'est pas apparue d'un coup. Elle provient de " ethniquement pur ", protoformule (p. 243). Elle devient d'un " mot des Albanais du Kosovo selon les Serbes ", un " mot des Serbes selon les instances politiques internationales "(p. 26os), se déplaçant géographiquement du Kosovo à la Bosnie-Herzégovine (procès 1983), pour arriver à la Croatie pure de Tudjman, et aux zones serbes pures; les accusateurs changent aussi : $1 /$ journalistes, Yougoslaves (Serbes, communistes) ; 2 / militants, instances politiques internationales, ce qui soulève le problème de l'information, la dépendance des sources (pp. 280-285).

Si l'apparition de la " formule " est très visible ${ }^{1}$, déterminer à qui imputer ce néologisme n'est pas évident. Puisque l'expression apparait d'abord dans la presse, on pourrait penser à un journaliste, mais il faudrait plutôt chercher une " personne qui évolue principalement

1 Même si deux occurrences « épuration ethnique n ont paru en France en 1941, il n'en a plus été fait mention avant 1992 (p. 375, n 264), " C'est bien l'expression qui est nouvelle, et non pas nécessairement les différents contenus qu'elle est à même d'accueillir ou les faits auxquels elle est capable de référer n (p. 287). 
dans la sphère du politique, de l'humanitaire, de la recherche, de la littérature ou encore qui intervient dans l'ensemble de ces domaines et qui est en outre préposée à l'expression d'une opinion ("intellectuel") " (p. 296). En fait, ce sont les sources de parole qui emploient l'expression que les récitants français traduisent par " purification " ou " nettoyage ethnique " (qui sont, comme dans le cadre de la proto-formule, ceux qui accusent). Elle est rapportée en France par ceux qui sont sur place et apparait donc comme un "néologisme par traduction " (p. 305)

A. Krieg-Planque doit également passer en revue les autres mots d'ordre : " ajouter la guerre à la guerre " (Mitterrand), "plus jamais ça ", " nous ne pourrions pas dire que nous ne savions pas " ${ }^{2}$, "l'Europe commence à Sarajevo "; les référents " techtniks " / " oustachis ", " $\mathrm{M} /$ musulman / bosniaque " / " bosno-serbe " (à l'usage mal stabilisé) ${ }^{3}$, les belligérants / victimes et bourreaux (qui introduit un point de vue moral dans la description des acteurs du conflit), les agresseurs / agressés (qui permet de faire des " Bosniaques ", des populations passives et victimisées, p. 190) ; la communauté internationale ; ainsi que d'autres désignants (urbicide, mémoricide, Milošević, de géopolitique [Yougoslavie, Macédoine, RSBiH, république $\mathrm{BiH}]$, langue, ...).

A. Krieg-Planque jette également un regard critique sur les textes qualifiés de " clefs" pour comprendre les conflits : le " Mémorandum ", la " Déclaration islamique ", la " Déroute de la vérité historique ". Bien qu'estimés fondamentaux, leur lecture est partiale, leur analyse embrouillée, leur traduction aléatoire.

Au printemps 1993, alors que les nationalistes croates se retournent clairement contre leurs anciens alliés musulmans (p.129), paraissent deux livres qui auront des incidences sur l'interprétation du conflit : le Livre noir : purification ethnique et crimes de guerre dans l'exYougoslavie, qui montre la culpabilité serbe dans les faits et Le nettoyage ethnique : documents historiques sur une idéologie serbe, qui montre la culpabilité serbe dans les intentions. Ils sont édités au moment où " des questionnements auraient pu surgir sur l'origine de l'expression" $"($ p. 132) 4 .

Dès août 1992, la formule passe d'une expression inédite à l'abstraction du concept (p. 252) ; elle s'est imposée à la plupart des commentateurs comme descripteur de la guerre (p. 385). Toutefois, il est paradoxal qu'un terme qui remporte le succès et se répande dans l'univers discursif soit nettement plus souvent qualifié de terme inadéquat que de terme adéquat (p. 448). Les raisons en sont probablement que les perceptions des causes des guerres, des responsabilités dans les guerres, et les " analyses " des événements ne sont pas toujours les mêmes. Comme l'a très bien montré l'auteur, chaque " désignant » des guerres yougosla-

\footnotetext{
${ }^{2}$ Ce mot d’ordre, bien qu'estimé rattaché à la Deuxième Guerre mondiale, fait en fait référence à celle de $1914^{-1918 ~(p . ~ 142) . ~}$

3 Toutefois, le langage étant porteur de sens, nous noterons, bien que cela ne soit pas le sujet de son travail, l'usage évolutif qu'elle fait de Musulman et Bosniaque, usage qu'elle explique pp. 178-179: le choix de "Bosniaque " aux dépends de "Musulman " est dirigé par la volonté d'effacer définitivement le critère religieux de la désignation ... D'une part, disons le, la désignation " Bosniaque n correspond mieux à la réalité des faits [comprend aussi des personnes de nationalité serbe ou croate qui ont fait le choix d'une Bosnie unitaire ...]. Cela soulève la question, hors sujet de toute façon, de qui décide qui peut et qui ne peut pas être bosniaque.
}

4 Tout comme le livre de P. Garde [Vie et mort de la Yougoslavie] a été salué par la presse comme " une clé indispensable pour décrypter le déferlement quotidien d'informations contradictoires n, sans tenir compte de la polémique dont il a fait l'objet (p. 133). 
ves est remis en cause, atténué, expliqué, cautionné, décrié, adopté, rejeté. L'utilisation de la formule varie encore entre descripteur d'un événement singulier et nouvelle catégorie de dénomination, même si cette dernière tendance semble se confirmer.

Travaillant sur un sujet sensible, A. Krieg-Planque s'est tenue à l'écart des partis-pris de ceux qui sont " pris dans leur moralité " et de ceux qui sont " coincés dans leur vision nationaliste ". C'est un sujet objectivé qu'elle a traité, un travail dense et minutieux qu'elle nous livre ici.

Patrick Michels

\section{Tomić [Yves] \\ La Serbie du prince Miloš à Milošević \\ Bruxelles / Bern / Berlin / Frankfurt / New York / Oxford /Wien : P. I. E. - Peter Lang, 2003, 165 p.}

L'ouvrage d'Yves Tomic comble un vide bibliographique important en France, puisqu'il retrace l'histoire de la Serbie (et non des Serbes, ce qui constitue une différence majeure) depuis le début du XIX ${ }^{e}$ siècle jusqu'à nos jours. La démarche de l'auteur, historien de formation, est de resituer les événements des deux dernières décennies du XX $\mathrm{X}^{\mathrm{e}}$ siècle en Serbie (et dans les Balkans) dans la longue durée de la " construction nationale serbe ", afin d'en permettre une meilleure compréhension.

Cinq chapitres retracent ainsi l'évolution de la Serbie : la construction nationale au XIX siècle ; la formation de l'idéologie nationale au XIX ${ }^{\mathrm{e}}$ siècle ; l'intégration nationale des Serbes dans la Yougoslavie ; la question nationale dans la Yougoslavie communiste ; du ressentiment à la guerre. La permanence de la question nationale, récurrente dans l'histoire récente serbe, s'expliquerait notamment par deux faits principaux : d'une part, la Serbie n'est jamais véritablement parvenue à consolider son propre Etat national ; de l'autre - et c'est extrêmement important pour comprendre les relations actuelles entre les Serbes de Serbie étroite et les autres Serbes (Bosnie-Herzégovine, Croatie, Kosovo) - les Serbes dans leur ensemble n'ont jamais été réunis au sein d'une même nation. Dans cet ouvrage, l'analyse des différents Etats yougoslaves depuis 1918 n'est pas négligée et l'on perçoit bien la progression de la position, parfois ambiguë, de la Serbie, ainsi que l'ouverture de la question serbe dans la Yougoslavie communiste.

Qualifié de " régime politique autoritaire " puis de dérive dictatoriale à partir de 1998, et non de totalitarisme, le système politique instauré par S. Milošević est finement étudié, bousculant un certain nombre d'idées reçues telles que le présumé soutien sans faille des citoyens serbes à leur président ou leur non résistance à sa politique, comme l'a prouvé la " révolution " du 5 octobre 2000.

l'ouvrage se termine sur un point d'interrogation quant à l'avenir de la Serbie et la capacité de ses nouveaux dirigeants à mener à bien une " transition " qui se révèle relativement chaotique, sur fond de problèmes territoriaux non résolus (Kosovo) et d'union bancale (Monténégro). 